\title{
Metastatic Alveolar Soft Part Sarcoma Responsive to Pazopanib after Progression through Sunitinib and Bevacizumab: Two Cases
}

\author{
William L. Read Felicia Williams \\ Winship Cancer Institute, Department of Hematology/Medical Oncology, \\ Emory University School of Medicine, Atlanta, Ga., USA
}

\section{Keywords}

Alveolar soft part sarcoma $\cdot$ Soft tissue sarcoma $\cdot$ Sunitinib $\cdot$ Pazopanib

\begin{abstract}
Alveolar soft part sarcoma (ASPS) is a rare soft tissue sarcoma with a propensity for lung metastases and indolent progression. ASPS is not responsive to chemotherapy, but there are case reports and small series describing benefit from drugs targeting the VEGF pathway. These drugs include sunitinib, cediranib and bevacizumab. There is no established secondline treatment for persons with ASPS progressing through first-line targeted therapy. We report two individuals with metastatic ASPS who obtained disease stabilization from sunitinib lasting over a year. After subsequent progression through sunitinib and second-line bevacizumab, both individuals again had disease response and subsequent stabilization from pazopanib.

(C) 2016 The Author(s)

Published by S. Karger AG, Basel
\end{abstract}

\section{Background}

Alveolar soft part sarcoma (ASPS) is a rare soft tissue sarcoma with a propensity for lung metastases and indolent progression [1]. ASPS is characterized by the $\operatorname{der}(17) \mathrm{t}(\mathrm{X} ; 17)$ (p11;q25) chromosomal translocation, which produces chimeric transcription factors that drive overexpression of genes involved in angiogenesis and metastasis [2]. Standard cytotoxic chemotherapy has a low response rate and no palliative benefit [3]. After a report by Stac- 


\section{Case Reports in Oncology}

chiotti et al. [4] describing responses and benefit associated with sunitinib treatment, this agent has become the first line for palliative treatment of metastatic ASPS. Sunitinib acts on several kinase targets and it is not known which ones are relevant for ASPS, but other agents also targeting VEGF are reportedly effective against ASPS; these are cediranib [5], a tyrosine kinase inhibitor (TKi) acting on VEGF receptors, and bevacizumab [6, 7], a monoclonal antibody binding VEGF.

Pazopanib is a TKi approved for the treatment of soft tissue sarcoma and renal cell carcinoma [8]. Pazopanib shares many tyrosine kinase targets with sunitinib, including those in the VEGF and PDGF pathways [9]. Here we report two individuals with metastatic ASPS whose tumors responded to pazopanib despite earlier progression through sunitinib and bevacizumab.

\section{Case 1}

A 31-year-old man noted an increasingly painful mass in his left calf. Imaging found a tumor together with lesions suspicious for metastases in the distal left tibia and the distal left femur. Biopsy revealed ASPS. Chest CT showed multiple lesions interpreted to be metastases. Brain MRI showed no lesions. He embarked on palliative treatment with sunitinib at $37.5 \mathrm{mg}$ daily. Three months later, his lung lesions were stable but his leg tumor continued to grow and became more painful. Sunitinib was held and he received a palliative course of radiation to the leg (30 Gy/10 fractions). Within a month of completing radiation, his tumor ulcerated and became infected and foul smelling. We hoped that this might be related to acute radiation toxicity, but over the ensuing 3 months, his leg worsened. Nine months after presentation, he underwent below-the-knee amputation with pathology confirming ASPS as well as tibial metastases. Two months postoperatively, it was clear that the metastasis in his distal femur was compromising his recovery and he underwent a palliative intralesional resection and plate stabilization of this lesion. He has had no further trouble from his leg or other bones and now walks with a prosthesis.

Chest imaging done 1 year after presentation described slow growth of pulmonary nodules as compared to prior imaging. It was thought this progression might be due to the several interruptions of sunitinib for surgery and radiation, but despite staying on sunitinib over the ensuing 16 months, the lung metastases continued to slowly grow. Sunitinib was discontinued and bevacizumab $10 \mathrm{mg} / \mathrm{kg} / 14$ days begun. Chest imaging 2 and again 5 months later described progression (fig. 1).

Bevacizumab was stopped, and 2 years after his initial presentation, he began pazopanib at $800 \mathrm{mg}$ daily. This was complicated by hypertension controlled with labetalol, diarrhea necessitating dose reduction to $600 \mathrm{mg}$, and hypopigmentation, which made him 'look like Santa Claus'. Imaging 2 months after pazopanib initiation showed a clear response of his many lung lesions followed by stability on subsequent CT scans. Ten months after initiation, he continues on pazopanib with stable disease and good quality of life.

Case 2

A 26-year-old woman noted pain and swelling in her proximal right arm. Imaging revealed a mass in the posterior compartment of the arm as well as innumerable lung lesions consistent with metastases. MRI showed no brain lesions. Biopsy revealed ASPS and she began sunitinib at $37.5 \mathrm{mg}$ daily. Toxicity included hand-foot syndrome (ultimately requiring dose reduction to $25 \mathrm{mg}$ daily), but imaging showed both primary tumor and metastases to have decreased in size on imaging 3 months after initiation. She continued on sunitinib for the ensuing 33 months with stable disease on imaging. At 33 months, because of worsening 


\section{Case Reports in Oncology}

neuropathic arm pain, sunitinib was held and palliative radiation (50 Gy/20 fractions) to the arm was administered, with sunitinib resumed afterwards. Arm pain and swelling initially worsened over several months before finally improving above baseline.

Following the treatment break for radiation, imaging showed lung nodules to have grown slightly. It was hoped that this was due to interruption of treatment, but over the ensuing year, the lung metastases continued to grow despite ongoing sunitinib. Forty-seven months from her initial presentation, sunitinib was discontinued and she began bevacizumab $7.5 \mathrm{mg} / \mathrm{m}^{2} / 14$ days. With this, she enjoyed a decrease in swelling in her right arm and consequent improvement in strength; we had not realized that sunitinib had been worsening the edema in her arm. CT of the chest 2 months later confirmed continuing progression and bevacizumab was discontinued. She embarked on cyclophosphamide and sirolimus, but tolerated this for only 2 months before discontinuing for worsened fatigue, anxiety, and shortness of breath.

Imaging confirmed progression of lung nodules, and 53 months after her initial presentation, she began pazopanib at $800 \mathrm{mg}$ daily. This too proved difficult to tolerate. She was admitted to the hospital for hypoxia and found to have a pulmonary embolism, which in retrospect was probably responsible for her earlier clinical decline. She began lowmolecular-weight heparin, and with this, her dyspnea improved over the subsequent weeks. After 4 days off, she resumed pazopanib at $400 \mathrm{mg}$ daily. Imaging 2 months later showed response in most lung nodules and subsequent follow-up imaging shows stable disease (fig. 2). At the time of this writing, she has been on pazopanib for 8 months with stable metastatic disease in the lung and improvement in arm pain. Her arm edema has not recurred.

\section{Discussion}

Pazopanib produced disease responses and subsequent stability in our two patients with ASPS. Activity of pazopanib against ASPS has been reported previously. Two participants with ASPS in a pediatric phase 1 of pazopanib were reported to have stable disease on treatment [10]. The poster presentation of an abstract by Dembla et al. [11] (though not the abstract) described a clinical response of lung metastases in a study participant with ASPS receiving pazopanib. An EORTC retrospective of the PALETTE study noted an individual with ASPS was among the $3.5 \%$ of study participants who stayed on pazopanib for over 2 years [12]. It is not surprising that pazopanib would have activity against a tumor type known to be sensitive to sunitinib, given the similar targets of the two TKi drugs. It is a little more surprising that one drug would be effective when a similar drug had failed. There is, however, considerable precedent for sequencing small-molecule drugs in the treatment of renal cell carcinoma (RCC), a more common cancer which, like ASPS, does not respond to cytotoxic chemotherapy.

Treatment principles established for RCC could guide the treatment of ASPS. A clinical trial described a 75\% disease control rate with pazopanib in persons with RCC progressing through sunitinib or bevacizumab [13]. Presumably, induced resistance to one drug can be sidestepped or negated by the different spectrum of action of the subsequent drug [14].

If ASPS and RCC share sensitivity and resistance mechanisms to TKis, the RCC playbook offers a possible salvage treatment for the future date when our two patients progress through pazopanib. Lenvatinib is a TKi with a spectrum of action similar to pazopanib and this year was approved by the FDA in combination with the mTORi everolimus for the salvage treatment of RCC previously treated with an anti-VEGF TKi. A phase 3 study showed 
that compared to either drug alone, the lenvatinib-everolimus combination significantly improved survival for study participants [15]. Many of these study participants had prior treatment with pazopanib or sunitinib. It is interesting to speculate about whether salvage treatment with lenvatinib and everolimus is worth exploring not just for pazopanib-responsive ASPS, but for pazopanib-responsive sarcoma in general.

\section{Ethics Statement}

The authors have no ethical conflicts to disclose.

\section{Disclosure Statement}

The authors have no conflicts of interest.

\section{References}

1 Jaber OI, Kirby PA: Alveolar soft part sarcoma. Arch Pathol Lab Med 2015;139:1459-1462.

-2 Covell DG, Wallqvist A, Kenney S, Vistica DT: Bioinformatic analysis of patient-derived ASPS gene expressions and ASPL-TFE3 fusion transcript levels identify potential therapeutic targets. PLoS ONE 2012; 7:e48023.

3 Reichardt P, Lindner T, Pink D, Thuss-Patience PC, Kretzschmar A, Dörken B: Chemotherapy in alveolar soft part sarcomas. What do we know? Eur J Cancer 2003;39:1511-1516.

4 Stacchiotti S, Tamborini E, Marrari A, et al: Response to sunitinib malate in advanced alveolar soft part sarcoma. Clin Cancer Res 2009;15:1096-1104.

5 Kummar S, Allen D, Monks A, et al: Cediranib for metastatic alveolar soft part sarcoma. J Clin Oncol 2013;31:2296-2302.

-6 Conde N, Cruz 0, Albert A, Mora J: Antiangiogenic treatment as a pre-operative management of alveolar soft-part sarcoma. Pediatr Blood Cancer 2011;57:1071-1073.

-7 Mir 0, Boudou-Rouquette P, Larousserie F, et al: Durable clinical activity of single-agent bevacizumab in a nonagenarian patient with metastatic alveolar soft part sarcoma. Anticancer Drugs 2012;23:745-748 Schutz FAB, Choueiri TK, Sternberg CN: Pazopanib: clinical development of a potent anti-angiogenic drug. Crit Rev Oncol Hematol 2011;77:163-171.

-9 Kitagawa D, Yokota K, Gouda M, et al: Activity-based kinase profiling of approved tyrosine kinase inhibitors. Genes Cells 2013;18:110-122.

10 Glade Bender JL, Lee A, Reid JM, et al: Phase I pharmacokinetic and pharmacodynamic study of pazopanib in children with soft tissue sarcoma and other refractory solid tumors: a children's oncology group phase I consortium report. J Clin Oncol 2013;31:3034-3043.

11 Dembla V, Fu S, Wheler J, et al: Outcomes of patients with advanced sarcoma enrolled in clinical trials of pazopanib in combination with histone deacetylase, mTOR, Her2, or MEK inhibitors. J Clin Oncol 2016;34(suppl):abstr 11057.

-12 Kasper B, Sleijfer S, Litière S, et al: Long-term responders and survivors on pazopanib for advanced soft tissue sarcomas: subanalysis of two European Organisation for Research and Treatment of Cancer (EORTC) clinical trials 62043 and 62072. Ann Oncol 2014;25:719-724.

-13 Hainsworth JD, Rubin MS, Arrowsmith ER, Khatcheressian J, Crane EJ, Franco LA: Pazopanib as secondline treatment after sunitinib or bevacizumab in patients with advanced renal cell carcinoma: a Sarah Cannon Oncology Research Consortium Phase II Trial. Clin Genitourin Cancer 2013;11:270-275.

-14 Ravaud A, Gross-Goupil M: Overcoming resistance to tyrosine kinase inhibitors in renal cell carcinoma. Cancer Treat Rev 2012;38:996-1003.

15 Motzer RJ, Hutson TE, Glen H, et al: Lenvatinib, everolimus, and the combination in patients with metastatic renal cell carcinoma: a randomised, phase 2, open-label, multicentre trial. Lancet Oncol 2015;16:1473-1482. 


\section{Case Reports in Oncology}

\begin{tabular}{l|l}
\hline Case Rep Oncol 2016;9:639-643 \\
\hline DOI: 10.1159/000450545 & $\begin{array}{l}\text { c } 2016 \text { The Author(s). Published by S. Karger AG, Basel } \\
\text { www.karger.com/cro }\end{array}$ \\
\hline
\end{tabular}

Read and Williams: Metastatic Alveolar Soft Part Sarcoma Responsive to Pazopanib after Progression through Sunitinib and Bevacizumab: Two Cases

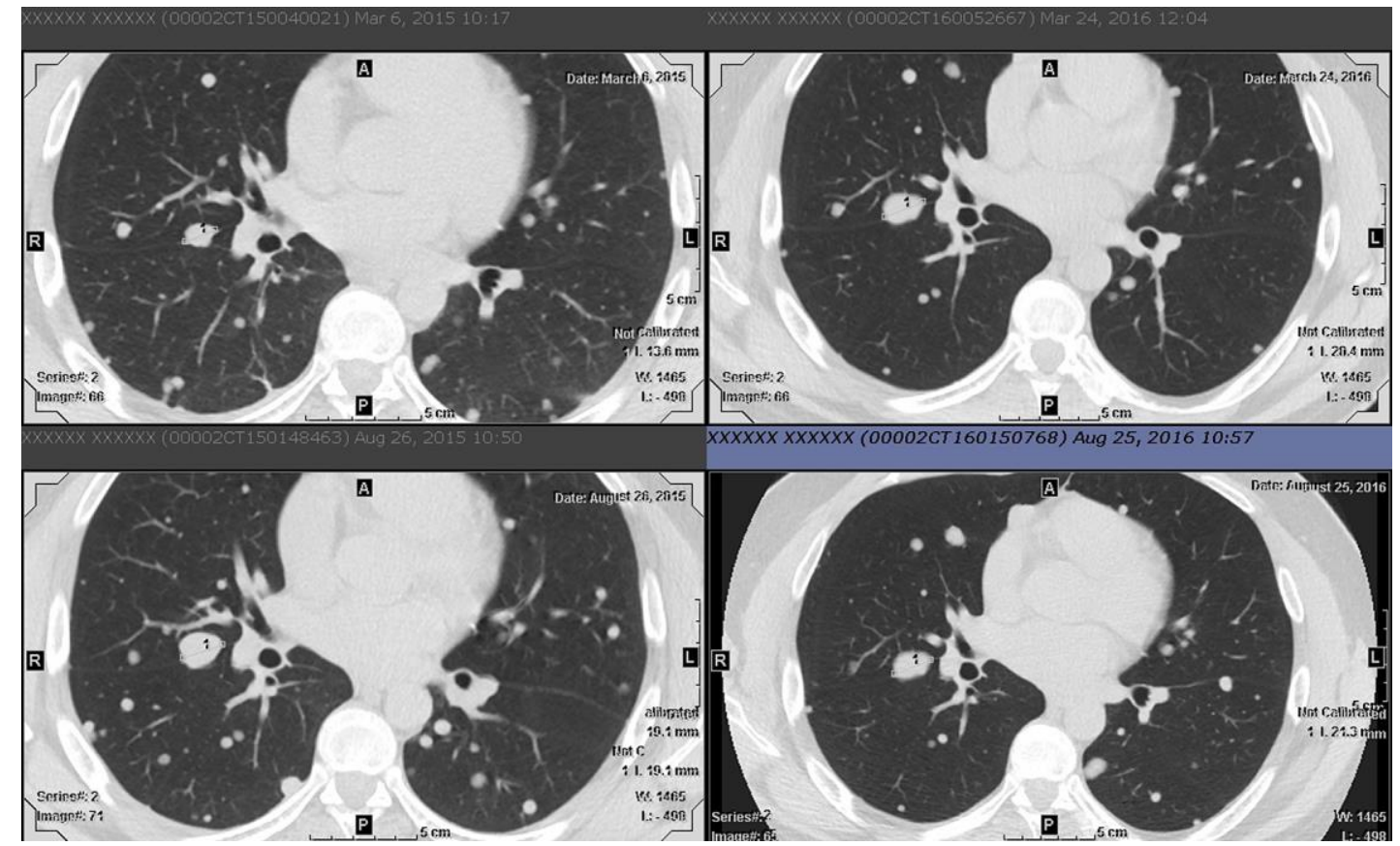

Fig. 1. Comparison of left images shows growth of the largest lung metastasis between March and August 2015 while on bevacizumab. On pazopanib, this lesion (and others) stopped growing or decreased in size between August 2015 and August 2016.

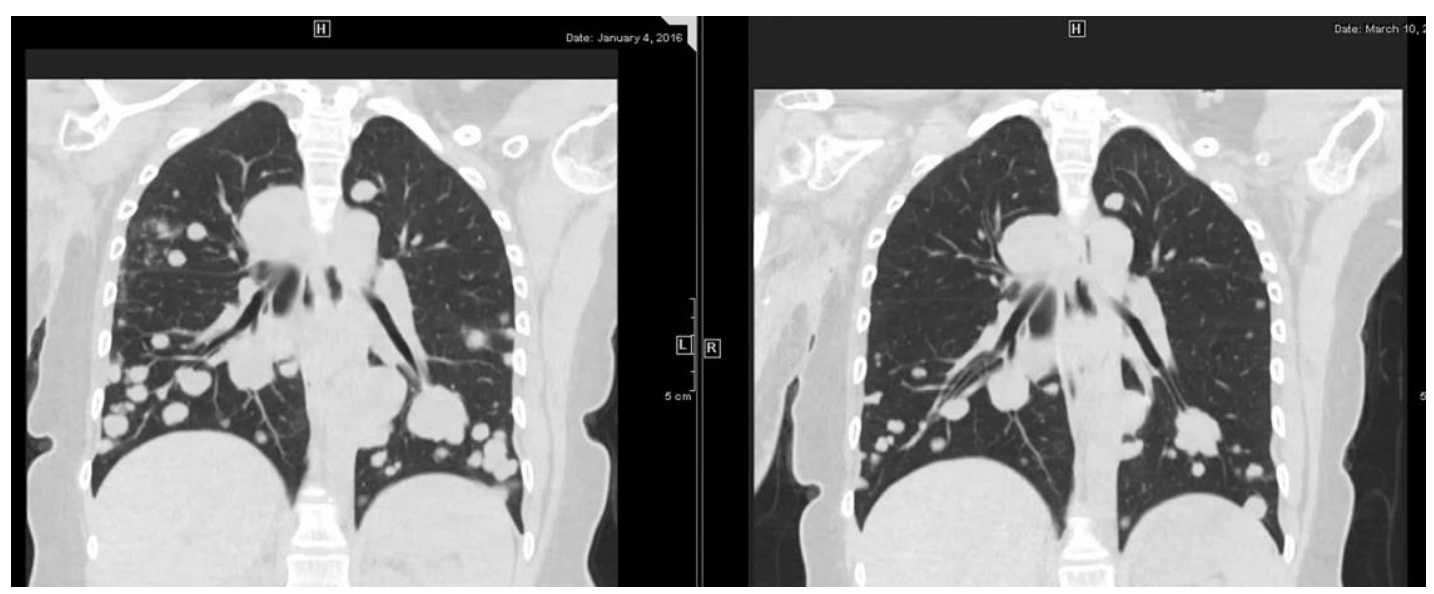

Fig. 2. After 2 months of pazopanib, the response of metastatic lesions in the lungs is obvious. 